\title{
Overview Scrollbar: A Scrollbar Showing an Entire Document as an Overview
}

\author{
Ko Mizoguchi, Daisuke Sakamoto, and Takeo Igarashi \\ The University of Tokyo \\ \{poohtokou, sakamoto, takeo\}@is.s.u-tokyo.ac.jp
}

\begin{abstract}
A scrollbar is the most basic function of a graphical user interface. It is usually displayed on one side of an application window when a displayed document is larger than the window. However, the scrollbar is mostly presented as a simple bar without much information, and there is still plenty of room for improvement. In this paper, we propose an overview scrollbar that displays an overview of the entire document on it and implemented four types of overview scrollbars that use different compression methods to render the overviews. We conducted a user study to investigate how people use these scrollbars and measured the performance of them. Our results suggest that overview scrollbars are more usable than is a traditional scrollbar when people search targets that are recognizable in overview.
\end{abstract}

Keywords: user interface, scrollbar, document navigation.

\section{Introduction}

People can access many documents easily by using computers, but they also face various problems inherent to electronic devices, so there is strong demand for improvements to electronic document interfaces. As an example of one such improvement, we redesigned the scrollbars of the computer application window. Traditional scrollbars are drawn compactly on one side of applications in order to not reduce the main document space. However, since the resolution of displays is getting higher nowadays, users would be able to see main documents in a comfortable size without the need for making scrollbars smaller.

In this paper, we propose an overview scrollbar, which is a wide scrollbar that shows an overview of an entire document. Users can browse through a document by using an overview of the document, which is placed on one side of the application window, and the scrollbar enables the users to quickly grasp an overview of a full document and jump to any location immediately by clicking the overview. We implemented four types of overview scrollbars (uniform scaling, vertical compression, fisheye, and perspective) that use different methods to compress the overviews. The first two scrollbars compress the overviews by using a constant ratio. When a document is very large, the compression ratio gets very high, so recognizing detailed contents from the overview may become difficult. The latter two scrollbars compress the overviews by spatially varying the ratios with focus and context areas. 
The compression ratio is low in the focus area and high in the context area. We conducted a user study to investigate the usability and the problems of the overview scrollbars. Results show that they were more usable than were the traditional scrollbars when the search targets can be recognized only by overviews; however, we found some problems, especially with the fisheye and perspective ones.

\section{Related Work}

Shneiderman suggested basic improvements to scrollbars in 1992, such as placing marks on scrollbars or drawing page numbers on sliders [1]. On the basis of these improvements, much research was done afterwards [2,3,4,5], but most of it was simple functions added to traditional scrollbars and did not mention drastic modifications such as our wider scrollbar.

Document visualization research has been done in the field of HCI. The Reader's Helper [6] is a document navigation system that uses Thumbar, which shows an overview of an entire document. Likewise, the Popout Prism [7] uses an overview panel to do so. These pieces of research used interfaces similar to our overview scrollbars, but they did not examine how to scale the overview in depth. Thus, we examined the scaling methods that are used in these pieces of research with our novel methods.

Hornbæk et al. conducted an experiment to compare the usability of linear, fisheye, and overview+detail interfaces on a reading environment [8,9]. Baudisch et al. implemented Fishnet, which is a web browser that uses a fisheye view [10], and compared the usability between that and other interfaces. The purpose of the former study was to investigate which interfaces were suitable for enhancing a reader's degree of comprehension, and the purpose of the latter was to investigate which interfaces perform well when readers search text based targets in a document by using highlighted annotations. In this paper, we investigate the performance of scrollbars when readers search targets in a large document by using its full overview.

\section{Overview Scrollbar}

We propose four types of scrollbars showing document overviews - uniform scaling, vertical compression, fisheye, and perspective. The area users see in the main view is highlighted on the scrollbar. Users can scroll the document to any location by left clicking or dragging on the scrollbar. Although the methods for compressing the overviews are different in these scrollbars, there are basic rules in compression.

The width of the overview scrollbar is limited to $20 \%$ of the application window. When the height of the document is too short, the overview is scaled so that the width fits its maximum size, preserving the aspect ratio. In this case, the height of the overview is shorter than that of the application, so the overviews are arranged on the top and nothing is drawn under the overviews. In the case that the height of the document is too large, the overview is compressed as follows. 
(a) Uniform Scaling Scrollbar: Figure 1(Left) and Figure 1(a) show the uniform scaling scrollbar. The aspect ratio of the overview is maintained regardless of the vertical size of the document. Thus, if the vertical size of the document is too large, the width of the overview may become too narrow.

(b) Vertical Compression Scrollbar: Figure 1(b) shows the vertical compression scrollbar. When the vertical size of the document is too large to maintain the aspect ratio of the overview, the overview is simply compressed vertically so that the height matches that of the application.

(c) Fisheye Scrollbar: Figure 1(c) shows the fisheye scrollbar. When the overview needs to be compressed vertically, a focus lens appears whose height is $70 \%$ that of the application. Within the lens, the compression ratio is low and constant with the preserved aspect ratio but gets higher as the distance from the lens increases. In this scrollbar, the width outside of the lens is compressed as well as the height. The user has to control two entities in this scroll bar: the focus lens and the knob (position of the contents shown in the main view). Users respectively move the knob and the lens by holding down the left or right buttons and dragging with the mouse. The user can also move them simultaneously by dragging with both mouse buttons held down.

(d) Perspective Scrollbar: Figure 1(d) shows the perspective scrollbar. This interface is equivalent to the fisheye scrollbar except that the width outside of the lens has a constant size, which is $20 \%$ of the application.

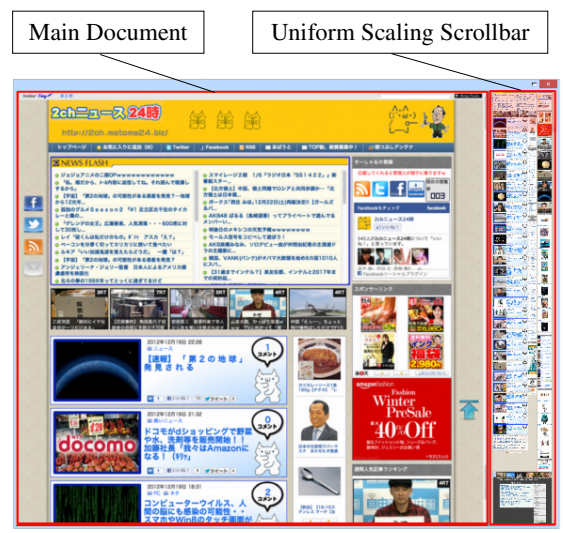

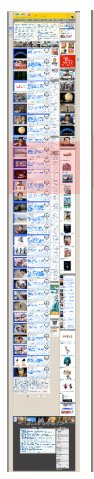

(a)

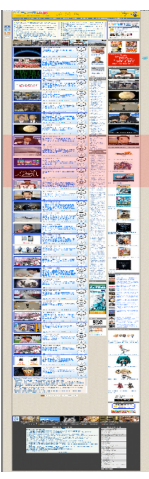

(b)

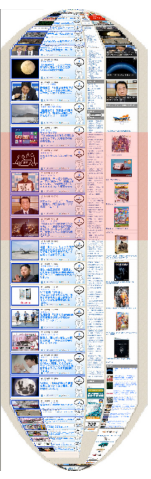

(c)

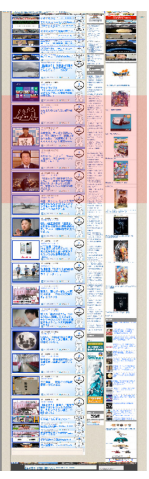

(d)

Fig. 1. Vertically large document with the uniform scaling scrollbar and the overview scrollbars: (a) uniform scaling, (b) vertical compression, (c) fisheye, and (d) perspective

\section{User Study}

We conducted a user study to investigate the performance and problems of the traditional scrollbar (called "standard scrollbar" below) and the overview scrollbars. We measured the task completion time, the distance scrolled by dragging the scrollbar, and the distance scrolled with the mouse wheel. Nine students participated in this study. All participants were computer science department students who were familiar with the standard scrollbar and had never used the overview scrollbar. 


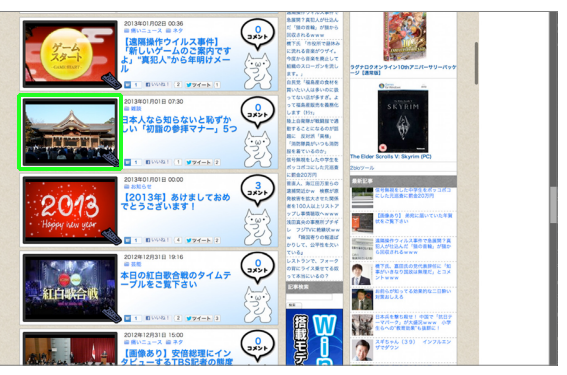

(a)

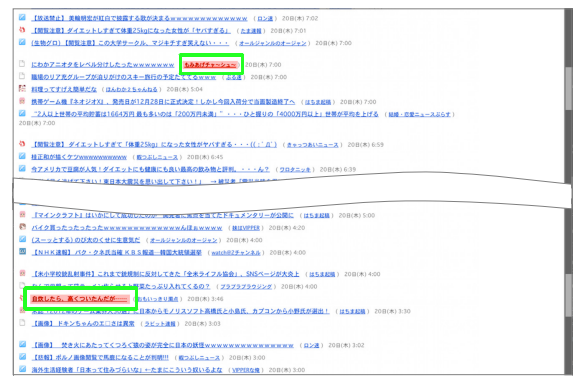

(c)

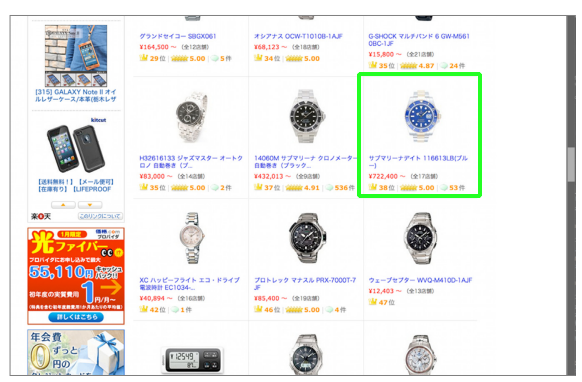

(b)

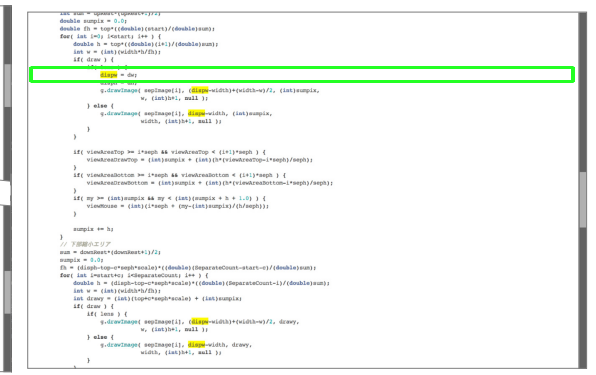

(d)

Fig. 2. Sample task execution screens. Search targets are surrounded by green rectangles. (a) Image search task, (b) Product search task, (c) Round trip task, and (d) Source code search task.

\subsection{Tasks}

Participants performed four types of tasks on each scrollbar. The same types of tasks were created so that they would all have the same of difficulty. All tasks required the participants to search for and click objects on a vertically long document. The documents were created beforehand as an image by processing the screen captures of real websites or source code.

(a) Image search task: Participants were asked to search an image on a large document (Figure 2(a)). The document size was about $1000 \times 7000$ pixels and had about 60 images on it. Before the task began, we specified an image of about $190 \times$ 140 pixels, whose instances appeared only once in the document.

(b) Product search task: Participants were asked to search for a product on a large document (Figure 2(b)). The document size was about $1000 \times 6500$ pixels and had 60 products on it. The products were arrayed in 3 columns and 20 rows and had one image representing the product and its title below the image. Before the task began, we specified a product that is unique in the document by using text. The text did not contain product specifications such as maker, model, or name but visual features such as color or form.

(c) Round trip task: Participants were asked to search for two links on a large document (Figure 2(c)). The document size was about $1000 \times 6000$ pixels and was 
mainly composed of text. There were two links highlighted in red and located near the top and bottom of the document. The participants were required to click these links six times mutually scrolling the documents up and down repeatedly.

(d) Source code search task: Participants were asked to search for a specified line on a large source code (Figure 2(d)). The source code contained approximately 300 lines written in Java. We chose words that appeared roughly 20 times in the code and highlighted them in yellow. The participants were asked to locate a line in which the word was used in a specific way, for example, a line where the highlighted word is substituted by a value or a word that appears in a conditional "if" statement.

\subsection{Questionnaire}

Participants were asked to answer a questionnaire after completing all trials. The questionnaire consisted of four Likert Scale questions (5 pts.) and three freeform questions (Table 1). In the Likert scale questionnaire, the participants were asked to answer a questionnaire item by using a five point scale ( 5 was the most positive).

Table 1. Questionnaire

\begin{tabular}{ccl}
\hline Type & $\#$ & Question \\
\hline & 1 & I felt that it was easy to get used to this method (get-used-to). \\
Likert & 2 & I thought that this method was easy to use (easy-to-use). \\
& 3 & I thought that this method was suitable for displaying documents (suitability). \\
& 4 & I want to use this method in practice (want-to-use). \\
\hline \hline \multirow{3}{*}{ Freeform } & 5 & Please write any good points and bad points in using this method. \\
& 6 & Please write any improvements for this method. \\
& 7 & Please write anything on your impressions or findings. \\
\hline
\end{tabular}

\subsection{Results}

\section{Performances}

A one-way within-subject ANOVA was conducted to compare the scrollbars. In the distance scrolled by dragging the scrollbar and with a mouse wheel, there were no statistical differences between all of them. However, there were significant differences in the task completion time. Figure 3(a) shows the average completion times for each task. In the round trip task, there were significant differences between the scrollbars $(F=12.75, p<.01)$. The vertical compression, uniform scaling, and perspective scrollbars were significantly faster than was the standard one $(p<.05)$. Likewise, the uniform scaling and the vertical compression ones were significantly faster than was the fisheye one $(p<.05)$, and the vertical compression one was significantly faster than was the perspective one $(p<.05)$. In the image search task, there were no significant differences. However, there was a tendency for the standard scrollbar to be faster than any of the others. In the source code search task, two participants got lost in the source code for a long time when using the standard and perspective scrollbars. Therefore, the average completion times for these two seemed very slow, but there were no statistical differences. 


\section{Preferences}

A one-way within-subject ANOVA was conducted in the questionnaire. Figure 3(b) shows the average scores of the participants for each task. In the first question, there were significant differences between the scrollbars $(F=4.33, p<.01)$. In pairwise comparisons, the uniform scaling scrollbar had a significantly higher score than did the fisheye one $(p<.05)$. In the second question, we also found significant differences between the scrollbars $(F=3.54, p<.05)$. In pairwise comparisons, the vertical compression scrollbar had a significantly higher score than did the standard one ( $p$ $<.05)$. In the third and forth questions, there were no significant differences. However, the standard scrollbar tended to be rated lower than any of the others.

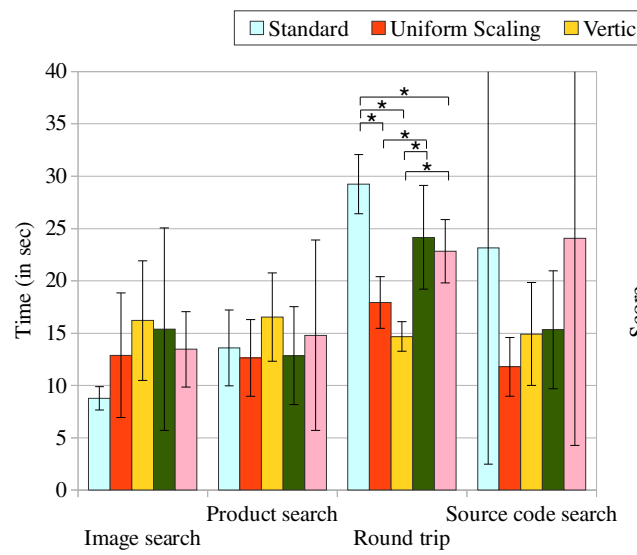

(a)

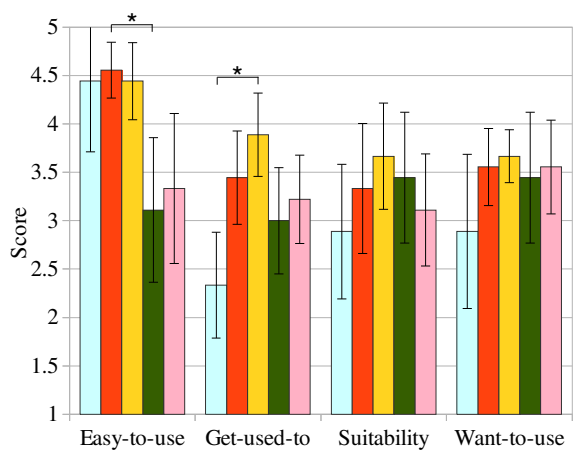

(b)

Fig. 3. (a) Average task completion times, (b) Average scores (error bars indicate $90 \%$ confidence intervals.)

\section{Freeform Comments}

We collected several comments for each scrollbar. For the standard scrollbar, some participants said, "It was very unstable to search for something in a document." $\mathrm{We}$ considered that they could not grasp an overview of the documents immediately, and they could not see where the targets were likely to be placed.

Some participants said, "I felt little difference between the uniform scaling and vertical compression scrollbars." The round trip task was easy with both of the scrollbars because participants could see where the red links were placed in one glance by looking at the overview even if it was highly compressed. However, most participants noticed that identifying detailed contents only by seeing the overview was difficult. Thus, in the image search and the product search tasks, these scrollbars might have been used simply as a wide scrollbar.

For the fisheye and perspective scrollbars, some commented that the images within the focus area were relatively easy to recognize, although it seemed to still be difficult to recognize contents completely. Likewise, almost all of the participants had difficulties in getting used to these scrollbars. They said, "The method of moving the focus lens by right-clicking was not intuitive and difficult to get accustomed to," and 
some of them commented that it would be better to integrate the right button function into the left button.

\section{Observations}

We observed how the participants performed the tasks. In the image search and product search tasks, most participants looked at the overview first and tried to find the target objects only from the overview. However, once they noticed that they could not recognize the contents only from the overview, they started to search targets mainly by looking at the main document. In the end, they used the overview scrollbars only for scrolling (although there were a few participants who could search images only from the overview when using the fisheye and perspective scrollbars.) Furthermore, we found that many participants seemed to have difficulties in getting used to the fisheye and perspective scrollbars. The successful participants tended to use these scrollbars by clicking the left and the right mouse buttons simultaneously, moving the focus lens and scrolling the document at the same time. They seemed to search for the targets efficiently.

\subsection{Summary of Results}

Our results suggest that there were significant differences in the average completion times in the round trip task. In this task, we found that the overview scrollbars were significantly faster than was the standard one. When the participants performed this task, unlike any other tasks, they were not required to recognize the detailed contents. Because of this feature, the advantages of the overview scrollbars that the participants can grasp an overview of the documents immediately and jump to the location they wanted immediately might have been emphasized. However, there were little differences except for in the round trip task. We guessed the reason for this result by checking the feedback and by observing the participants. Many participants suggested that the overview scrollbars (even the fisheye and perspective ones) were not powerful enough to let them completely grasp the detailed contents in a document. Therefore, as we observed, the participants tended to cope with the tasks by simply seeing the main document after a short trial of identifying the contents only with the overviews. Or rather, such a meaningless trial might produce some delays in image search and product search tasks, which might be one of the reasons why the standard scrollbar seemed to perform better.

\section{Conclusion}

We presented four types of overview scrollbars, conducted a user study, and analyzed study results. The result of the study and the feedback from the participants revealed to us many aspects for improvement in usability and for solving the problems of the overview scrollbars. First, we think that uniform scaling and vertical compression are already acceptable methods for document navigation systems because they marked a relative superior result to the other scrollbars both in performances and evaluations. Second, the user interfaces of the fisheye and perspective scrollbars were not perfect. If these two are implemented in a more sophisticated way, these methods might become more useful interfaces for navigating documents. 
However, there are some limitations on this research. First, our user study was conducted in a small sample group so that a formal user study is necessary to verify usability of the interface. Likewise, comparison studies between not only the scrollbars but also the scrollbars and the other types of methods that were useful when showing long documents such as Perspective-Drag or Zoom-and-Drag mentioned in [11]. Finally, we didn't use eye-tracking systems to analyze the user's behavior through the study. How users switch visual attentions between scrollbars and contents and how they interact with the scrollbars are both interesting. By quantifying these interactions, there may arise valuable results and discussions.

\section{References}

1. Shneiderman, B.: Designing the user interface: strategies for effective human-computer interaction. Addison-Wesley Longman Publishing Co., Inc., Boston (1986)

2. Björk, S.: The scrollsearcher technique: Using scrollbars to explore... In: Proc. of Interact 2001, Eighth IFIP TC.13 Conference on Human-Computer Interaction, pp. 745-746. IOS Press (2001)

3. Byrd, D.: A scrollbar-based visualization for document navigation. In: Proceedings of the Fourth ACM Conference on Digital Libraries, DL 1999, pp. 122-129. ACM, New York (1999)

4. Alexander, J., Cockburn, A., Fitchett, S., Gutwin, C., Greenberg, S.: Revisiting read wear: analysis, design, and evaluation of a footprints scrollbar. In: Proceedings of the SIGCHI Conference on Human Factors in Computing Systems, CHI 2009, pp. 1665-1674. ACM, New York (2009)

5. Laakso, S.A., Pekka Laakso, K., Saura, A.J.: Improved scroll bars. In: Proceedings of Human Factors in Computing Systems, CHI 2000, pp. 97-98. ACM Press (2000)

6. Graham, J.: The reader's helper: a personalized document reading environment. In: Proceedings of the SIGCHI Conference on Human Factors in Computing Systems, CHI 1999, pp. 481-488. ACM, New York (1999)

7. Suh, B., Woodruff, A., Rosenholtz, R., Glass, A.: Popout prism: adding perceptual principles to overview+detail document interfaces. In: Proceedings of the SIGCHI Conference on Human Factors in Computing Systems, CHI 2002, pp. 251-258. ACM, New York (2002)

8. Hornbæk, K., Frökjær, E.: Reading of electronic documents: the usability of linear, fisheye, and overview+detail interfaces. In: Proceedings of the SIGCHI Conference on Human Factors in Computing Systems, CHI 2001, pp. 293-300. ACM, New York (2001)

9. Hornbæk, K., Frökjær, E.: Reading patterns and usability in visualizations of electronic documents. ACM Trans. Comput.- Hum. Interact. 10(2), 119-149 (2003)

10. Baudisch, P., Lee, B., Hanna, L.: Fishnet, a fisheye web browser with search term popouts: a comparative evaluation with overview and linear view. In: Proceedings of the Working Conference on Advanced Visual Interfaces, AVI 2004, pp. 133-140. ACM, New York (2004)

11. Guiard, Y., Du, Y., Chapuis, O.: Quantifying degree of goal directedness in document navigation: application to the evaluation of the perspective-drag technique. In: Proceedings of the SIGCHI Conference on Human Factors in Computing Systems, CHI 2007, pp. 327336. ACM, New York (2007) 